\title{
OPERATION OF ALADDIN AT LOWERED EMITTANCE*
}

\author{
J.J. Bisognano, R.A. Bosch, D.E. Eisert, M.A. Green, K.J. Kleman, W.S. Trzeciak, Synchrotron \\ Radiation Center, University of Wisconsin-Madison, Stoughton, WI 53589 USA
}

\begin{abstract}
To increase the available photon flux density for users, alternate lattice tunings of the Aladdin synchrotron light source have been developed with horizontal emittances significantly lower than the present value of $127 \pi \mathrm{nm}$ rad. Reduction of the horizontal emittance by a factor of three has been obtained experimentally. When the fourth harmonic Landau cavity is used to lengthen the bunch, the observed beam lifetime with the new lattice is not significantly changed from that of the existing lattice. The present goal is to achieve a factor of four horizontal and a factor of two vertical emittance reduction routinely. Progress in making this new configuration fully operational is discussed, including the use of quadrupole shunts with correction software for betafunction and dispersion correction, understanding of a mode coupling instability in higher-harmonic RF systems, RF clearing of ions, and fabrication of new optical monitoring stations.
\end{abstract}

\section{INTRODUCTION}

The operation of the Synchrotron Radiation Center (SRC) Aladdin storage ring at lower emittance would increase the available flux focused through 19 of 21 monochromator entrance slits and increase the flux density available from most beamlines [1]. Decreased horizontal beam size would be especially useful to our user community. Spatial coherence in one of the undulator lines at a typical photon energy of $10 \mathrm{eV}$ would be improved by nearly an order of magnitude. The simplest and least expensive route to low emittance calls for preservation of the present magnet locations, but with new quadrupole and sextupole powerings. Solutions with significantly lower natural emittance, approaching the theoretical minimum emittance, were found. These solutions allow continued use of high-efficiency, offenergy injection into a high dispersion straight section of the present Aladdin lattice. Furthermore, issues such as momentum aperture, bunch stability, and lifetime can be addressed experimentally without jeopardizing the highly reliable routine operation in the existing configuration.

\section{LATTICE RETUNING}

The low-emittance lattices were obtained [2] using the matching capabilities of the MAD [3] program to transform the present lattice (which will be referred to as

* This work is supported by the NSF under Award No. DMR-0084402
"Aladdin") by varying the strengths of the nine quadrupole families and two sextupole families. The matching routine attempts to satisfy a set of constraints in a least-square sense, where the relative weighting of constraints may be modified by the user. To achieve a significant emittance decrease from Aladdin, we varied the quadrupole strengths such that, in MAD notation, $\left|K_{1}\right|<7.5$ and sextupole strengths such that $\left|K_{2}\right|<87.5$, allowing $800 \mathrm{MeV}$ operation with no new magnets. To achieve low emittance, we used the matching constraints of $\eta=0$ at bending-magnet entrances and exits. The geometry of Aladdin is a square with three 30-degree bends on each corner. In its original design, the three bends were constrained to offer comparable beam sizes. This constraint was dropped in obtaining the lower emittance configurations. However, because of the lowered emittance all bending magnets sources achieve better performance. Dispersion in the four undulator straight sections is reduced significantly and results in further decrease in beam size. Since the momentum compaction, $\alpha$, roughly obeys [4]

$$
\alpha \approx \frac{J_{x} \rho_{\mathrm{o}} v_{x}}{C_{q} R \gamma_{\mathrm{o}}^{2}} \varepsilon_{x},
$$

the low-emittance lattices typically have a momentum compaction an order of magnitude less than Aladdin, resulting in a bunch length about one-third that of Aladdin for a given RF voltage. This tends to reduce longitudinal beam stability and lifetime, but as discussed later, these effects can be managed with the bunch lengthening Landau cavity.

We constrained the tunes $\left(v_{x}, v_{y}\right)=(7.13,7.20)$ to the Aladdin values to allow injection at the nominal $108 \mathrm{MeV}$ into the existing Aladdin lattice, followed by slewing to the new lattices at the full energy of $800 \mathrm{MeV}$. For $\beta_{\max }=$ $15,20,25$, or $30 \mathrm{~m}$, we constrained the betatron functions by $\beta_{\mathrm{x}}, \beta_{\mathrm{y}}<\beta_{\max }$ to create four lattices that display differing trade-offs between low emittance and long lifetime. Effective chromaticity correction with $\left|\mathrm{K}_{2}\right|<$ 87.5 was obtained.

The lattices generated by these matching constraints are denoted L15, L20, L25, and L30, (e.g., Low-emittance 15 meter betatron function maximum). These lattices have natural horizontal emittance, $\varepsilon_{\mathrm{xo}}$, ranging from $8.1 \pi \mathrm{nm}$ $\operatorname{rad}$ for L30 to $41.0 \pi \mathrm{nm}$-rad for L15. In comparison, the Aladdin calculated natural emittance is $127 \pi \mathrm{nm}$-rad, so that the low-emittance lattices offer an emittance reduction of $68-94 \%$. Although the SRC magnet 
placement was not originally designed for these lowemittance lattices, the emittance of the L30 lattice equals the minimum emittance obtainable with 30 degree bending magnets and $800 \mathrm{MeV}$ ring energy from a Chasman-Green (double-focusing achromat) lattice, for which $\min \left(\varepsilon_{\mathrm{xo}}\right)=2.48 \times 10^{-14} \gamma^{2} \phi_{\mathrm{B}}{ }^{3} \pi \mathrm{m}$-rad, where $\gamma$ is the relativistic factor and $\phi_{\mathrm{B}}$ the deflection in a bending magnet (in radians). This suggests that further emittance reduction may be elusive.

For a given RF-voltage, the bunch length is roughly proportional to the square root of the emittance. With bunch lengths of $\sim 1 / 3$ the Aladdin value, the lowemittance lattices have reduced Touschek lifetimes. However, the installed fourth-harmonic cavity is expected to lengthen the bunch by a factor of $\sim 4$ (versus 2.3 for Aladdin) at the nominal energy spread. In addition, because of the smaller dispersion and momentum compaction of the low emittance lattices, the momentum aperture associated with the physical aperture and bucket height, respectively, are greater than in the present Aladdin. As a result, a lattice with a horizontal emittance

\begin{tabular}{|c|c|c|c|c|c|}
\hline & Aladdin & L15 & L20 & L25 & L30 \\
\hline $\begin{array}{l}\text { natural emittance }(\pi \mathrm{nm}- \\
\mathrm{rad})\end{array}$ & 127 & 41.0 & 24.2 & 14.6 & 8.1 \\
\hline momentum compaction & 0.034 & 0.0048 & 0.0038 & 0.0030 & 0.0036 \\
\hline $\max \left(\beta_{\mathrm{x}}\right)$ (meters) & 12 & 15 & 20 & 25 & 30 \\
\hline $\max \left(\beta_{\mathrm{y}}\right) \quad$ (meters) & 18 & 15 & 20 & 25 & 30 \\
\hline bunch length (mm) & 76 & 30 & 27 & 24 & 26 \\
\hline center of LSS: $\beta_{\mathrm{x}}(\mathrm{m})$ & 6.6 & 1.9 & 1.8 & 1.6 & 1.9 \\
\hline center of LSS: $\beta_{\mathrm{y}} \quad(\mathrm{m})$ & 4.2 & 2.5 & 1.8 & 1.4 & 1.9 \\
\hline center of LSS: $\eta_{x} \quad(m)$ & -0.67 & 0.19 & 0.25 & 0.30 & 0.32 \\
\hline $\begin{array}{llll}\text { bending magnet } 1: & \sigma_{x} \\
(\mu \mathrm{m})\end{array}$ & 506-564 & $138-170$ & $90-158$ & $71-151$ & $49-130$ \\
\hline $\begin{array}{l}\text { bending magnet 2: } \sigma_{x} \\
(\mu \mathrm{m})\end{array}$ & $467-485$ & $189-227$ & $\begin{array}{l}139- \\
179\end{array}$ & $\begin{array}{l}106- \\
141\end{array}$ & 70- 115 \\
\hline $\begin{array}{l}\text { bending magnet } 3: \sigma_{x} \\
(\mu \mathrm{m})\end{array}$ & $539-543$ & $138-195$ & 95- 135 & $65-93$ & $45-74$ \\
\hline $\begin{array}{l}\text { bending magnet } 1: \sigma_{\mathrm{y}} \\
(\mu \mathrm{m})\end{array}$ & $65-86$ & $24-31$ & $21-31$ & $11-16$ & 7.5- 9.3 \\
\hline $\begin{array}{l}\text { bending magnet } 2: \sigma_{\mathrm{y}} \\
(\mu \mathrm{m})\end{array}$ & $73-94$ & $49-64$ & $43-57$ & $37-50$ & $30-41$ \\
\hline $\begin{array}{l}\text { bending magnet } 3: \sigma_{y} \\
(\mu \mathrm{m})\end{array}$ & $68-85$ & $58-70$ & $50-61$ & $46-54$ & $38-45$ \\
\hline LSS middle: $\sigma_{x}(\mu \mathrm{m})$ & 960 & 294 & 239 & 209 & 196 \\
\hline LSS middle: $\sigma_{\mathrm{y}}(\mu \mathrm{m})$ & 64 & 31 & 21 & 14 & 12 \\
\hline
\end{tabular}

Table I: Lattice properties computed by MAD for 800 $\mathrm{MeV}$ ring energy and $1 \%$ emittance coupling. one third that of Aladdin and an unchanged vertical emittance has a Touschek lifetime that is $30 \%$ larger, assuming that good dynamic aperture and perfect lattice function correction is obtained. The source dimensions in the low-emittance lattices are about $25-67 \%$ of the Aladdin values. For the Aladdin lattice and the lowemittance lattices, Table I summarizes the lattice properties computed by MAD.

\section{MOMENTUM APERTURE}

The dynamic apertures were studied at the same tunes as Aladdin by tracking for 1000 turns an electron which has an initial horizontal or vertical position offset in the middle of the first long straight section (LSS). The maximum offsets $\left(x_{\max }\right.$ and $\left.y_{\max }\right)$ that could be tracked 1000 turns (without deviating $1 \mathrm{~m}$ from the design orbit) define the dynamic aperture at the LSS center. By including round quadrupole apertures (radius $=32 \mathrm{~mm}$ ) in the center of all quadrupoles, and rectangular undulator apertures (half-width $=30 \mathrm{~mm}$, half-height $=9.5 \mathrm{~mm}$ ) at the beginning, middle, and end of the long straight sections, the physical aperture was obtained. In all cases studied, the physical aperture was significantly smaller than the dynamic aperture, so that dynamic aperture effects are not expected to limit the machine performance at the tunes studied.

\section{FEASIBILITY EXPERIMENT}

Experimental studies of the mid-performance L20 lowemittance $\left(\varepsilon_{\mathrm{x}} \approx 25 \mathrm{~nm}-\mathrm{rad}\right)$ lattice were carried out and indicate clearly that substantial reduction in beam size is both feasible and operationally sound. After injection and acceleration to $800 \mathrm{MeV}$, the Aladdin ring quadrupoles were slewed by the control system to the new values with essentially no loss of current. Up to $250 \mathrm{~mA}$, a standard fill current, was successfully stored in the low emittance configuration. Horizontal beam size observed with optical monitors at dipole magnets decreased by a factor of two to four, and higher photon flux density was observed on a reference sample in the MEPHISTO spectromicroscope mounted on the HERMON beam line. Observed beam jitter was unchanged from the standard operational mode. A factor of two increase in flux density was also observed on the PGM undulator beamline. It is estimated that the emittance decreased from about $127 \pi$ $\mathrm{nm}$-rad to $35 \pi \mathrm{nm}$-rad, which may be due to uncorrected quadrupole errors.

\section{COLLECTIVE EFFECTS}

At lower emittance, the beam density increases and a number of collective phenomena can be exacerbated. An important finding of these studies is that high-density effects are well tolerated. In a passive mode, the fourth harmonic cavity successfully stabilized coupled bunch instabilities at the full $250 \mathrm{~mA}$ and lengthened the bunch. 
With the lengthened bunch, current-lifetimes equaled that achieved in the standard, high-emittance mode (1000 mAh) with the horizontal emittance reduced by a factor of three. In the active mode, the fourth harmonic cavity was found unstable at beam currents greater than $100 \mathrm{~mA}$. Analytic modeling and simulations suggest that the instabilities result from fast mode coupling between the dipole and quadrupole Robinson modes [5]. Spectral observations confirm this hypothesis. Work continues on specification of the necessary RF system modifications to allow the more robust active operation of the fourth harmonic system. Increased feedback loop gain is a likely solution. A separate feedback system is also being considered to suppress the longitudinal instability.

With lower momentum compaction factors, the microwave instability is a concern. In particular, any substantial increase in energy spread could reduce the effectiveness of undulators at high harmonics. Measurements of energy spread as a function of single bunch current are consistent with a broadband impedance of about $10 \mathrm{ohms}$. Since observed synchrotron tune shifts are small (consistent with $1 \mathrm{ohm}$ reactive), Aladdin is not in the long bunch regime of the microwave instability although the nominal bunch length is considerably larger than the beam pipe radius. With the estimated $10 \mathrm{ohms}$ impedance and with the use of the Landau cavity, rms energy spread should remain below 0.001 at full current. This is consistent with operation of our newest undulator at its designed ninth harmonic.

\section{INFRASTRUCTURE UPGRADES}

To provide better diagnostics to correct coupling, five new optical diagnostic stations will replace the current three of which only one gives full beam profile with rotation. In each new setup, a water-cooled $\mathrm{Si}$ mirror deflects about $15 \mathrm{mrad}$ into the light optics line. A lens system focuses light on a CCD camera. Frame grabber and analysis software provides position, size and rotation at about a $10 \mathrm{~Hz}$ rate using a $10 \mathrm{~nm}$ bandpass at $400 \mathrm{~nm}$ to optimize resolution. The system is capable of beam splitting for another detector such as a position sensitive photodiode to get information on fast motions.

As a serendipitous outgrowth of attempts to measure electron beam polarization, it was found that rf kicks can have a significant impact on ion trapping in Aladdin. About $1 \mathrm{~W}$ at $7.5 \mathrm{MHz}$ is applied to a pair of vertical striplines $\sim 0.4 \mathrm{~m}$ long at a high vertical beta point. In addition, the standard dc clearing-electrode voltages are applied throughout the accelerator. The drive frequency is $\sim 50 \mathrm{kHz}$ above the vertical betatron sideband of the second rotation harmonic. The frequency is chosen to be close enough to shake the ions without overly shaking the beam. Substantial clearing is possible with sub micrometer motions of the beam. Other rotation harmonics were tried but the second gave minimum tune spread. Frequencies are in approximate agreement with simple ion trapping theory [6]. The observed vertical tune spread is decreased by roughly a factor of two by rf clearing. The rf clearing is most dramatic when the accelerator vacuum is sub-optimal as occurs when beampipe or beamline conditioning is necessary or after a machine shut down period. Without rf clearing, vertical beam size can increase by $10-20 \%$ or insertion device compensation can fail. Radio-frequency clearing was found to return the accelerator close to normal conditions.

\section{TOWARD ROUTINE OPERATION}

A significant deviation from four-fold symmetry was also observed during these machine studies, including anomalous horizontal and vertical dispersion. This was not unexpected and is due to percent-level errors in quadrupole magnetic fields that had been analyzed with the code LOCO [7]. It appears that these dispersion errors reduce the momentum acceptance of the ring by roughly $30 \%$ by inducing scraping on the physical aperture. Touschek scattering calculations with the code ZAP [8] indicate that that this may be reducing beam lifetimes by roughly $40 \%$. In addition, attempts to operate the storage ring below $35 \mathrm{~nm}$-rad have so far been unsuccessful and may also be due to the uncorrected dispersion. Rather simple and inexpensive shunts have recently been installed, which will provide beam optics corrections. The expected lifetime improvements and $H$-function correction should allow further reduction in beam emittance. The present goal is to be operational for users at the end of 2001 with a factor of four horizontal emittance reduction and a factor of two vertical emittance reduction. In the long term, some of the limiting apertures may be opened to increase the momentum acceptance of the ring to maximize beam lifetime.

\section{ACKNOWLEDGMENTS}

We thank Mary Severson and Pupa DeStasio for the measurements of flux density on the PGM and HERMON beamlines, respectively.

\section{REFERENCES}

[1] C. Olson (private communication).

[2] R. A. Bosch and W. S. Trzeciak, SRC-170 (1997).

[3] H. Grote and F. C. Iselin, CERN/SL/90-13 (1990).

[4] M. Sands, SLAC-121 (1970).

[5] R .A. Bosch, K .J. Kleman and J. J. Bisognano, "Influence of the Momentum Compaction upon Landau-Cavity Stability," (this conference).

[6] A. Poncet, CERN 90-04, p. 74 (1990).

[7] J. Safranek, Nucl. Instrum. Methods Phys. Res., Sect. A 388, 27 (1997).

[8] M. Zisman, S. Chattopadhyay, and J. Bisognano, LBL-21270 (1986). 